\section{Autonomia financeira em estabelecimentos públicos e privados de saúde no Brasil}

\author{
Autonomy for financial management in public and \\ private healthcare facilities in Brazil
}

\section{Autonomía financiera de establecimientos públicos y privados de salud en Brasil}

\author{
1 Escola Nacional de Saúde \\ Pública Sergio Arouca \\ Fundação Oswaldo Cruz, Rio \\ de Janeiro, Brasil. \\ 2 Secretaria Municipal de \\ Saúde do Rio de Janeiro, Rio \\ de Janeiro, Brasil. \\ 3 Instituto Brasileiro de \\ Geografia e Estatística, Rio de \\ Janeiro, Brasil. \\ 4 Instituto de Pesquisa \\ Clínica Evandro Chagas, \\ Fundação Oswaldo Cruz, Rio \\ de Janeiro Brasil. \\ Correspondência \\ M. A. B. Santos \\ Escola Nacional de Saúde \\ Pública Sergio Arouca, \\ Fundação Oswaldo Cruz. \\ Av. Brasil 4036, sala 901, \\ Rio de Janeiro, $R$ \\ 21040-361, Brasil. \\ angelicabsantos@uol.com.br
}

\begin{abstract}
Autonomy in financial management is an advantage in public administration. A $2009 \mathrm{Na}-$ tional Healthcare Facility Survey showed that 3.9\% of Brazil's 52,055 public healthcare facilities had some degree of financial autonomy. Such autonomy was more common in inpatient facilities (17.8\%), those managed by State governments (26.3\%), and in Southern Brazil (6.6\%). Autonomy was mainly partial (for resources in specific areas, relating to small outlays, consumables and capital goods, and outsourced services or personnel). $74.3 \%$ of 2,264 public facilities with any financial autonomy were under direct government administration Financial autonomy in public healthcare facilities appears to be linked to local political decisions and not necessarily to the facility's specific legal and administrative status. However, legal status displays distinct scopes of autonomy those under direct government administration tend to be less autonomous, and those under private businesses more autonomous; $85.8 \%$ of the 45,394 private healthcare facilities reported that they were financially autonomous.
\end{abstract}

Health Services Administration; Health Services Research; Financial Management
Maria Angelica Borges dos Santos 1,2

Fátima Carvalho Madeira 3

Sonia Regina Lambert Passos 4

Felipe Bakr 1

Klívia Brayner de Oliveira 3

Marco Antonio Ratzsch de Andreazzi 3

\section{Resumo}

Autonomia financeira é um diferencial positivo na gestão pública, sendo referida na Pesquisa de Assistência Médico-Sanitária (2009) por 3,9\% dos 52.055 estabelecimentos públicos de saúde. Foi mais frequente em estabelecimentos públicos com internação $(17,8 \%$ do total), da administração estadual (26,3\%) e da Região Sul (6,6\%) e, em geral, era parcial (apenas para algumas áreas, como despesas emergenciais, material de consumo e permanentes, serviços de terceiros e pessoal). Quanto à natureza jurídica, 83,7\% dos 2.011 estabelecimentos públicos que referiram alguma autonomia são da administração direta. A atribuição de autonomia financeira a estabelecimentos públicos de saúde parece ser definição política local, que não se prende necessariamente a mudanças de natureza jurídica. Ainda assim, confirmou-se um espectro crescente de maior autonomia segundo natureza jurídica, em que estabelecimentos da administração direta da saúde são os menos autônomos $e$ as sociedades empresariais as mais autônomas; 85,8\% dos 45.394 estabelecimentos privados tinham alguma autonomia financeira.

Administração de Serviços de Saúde Administração Financeira; Pesquisa sobre Serviços de Saúde 
Na administração pública, autonomia financeira significa "capacidade de gestão e aplicação dos recursos destinados a prover as atividades e serviços do órgão titular da dotação... pressupõe a existência de dotações que possam ser livremente administradas, aplicadas e remanejadas pela unidade orçamentária a que forem destinadas" 1 (p. 56).

Autonomia financeira é considerada um diferencial positivo na gestão pública ${ }^{2}$. Baixa autonomia financeira tem impactos negativos sobre profissionalização e planejamento de compras e contratos, elementos centrais para o funcionamento eficiente da rede pública de saúde ${ }^{3}$.

Para permitir que atividades caracterizadas como de serviço público ou interesse público tivessem maior autonomia financeira, foram criadas, na década de 1960, naturezas jurídicas alternativas à administração pública direta, como as autarquias. O debate sobre naturezas jurídicas alternativas intensificou no Brasil com o Plano Bresser 4 na década de 1990. A natureza jurídica de organizações habilitadas à prestação direta de serviços públicos expandiu, passando a incluir, além das públicas, organizações privadas sem finalidades lucrativas, caracterizadas como organizações sociais e organizações sociais de interesse público. Ainda assim, a grande maioria dos estabelecimentos do SUS pertence à administração direta da saúde.

Várias secretarias de saúde têm investido na descentralização orçamentária, conferindo a seus estabelecimentos alguma autonomia financeira, ainda que restrita em volume de recursos e escopo. No menor grau de autonomia, estabelecimentos de saúde da administração direta podem dispor de pequenos fundos, em geral valores compatíveis com compras emergenciais. Em graus mais sofisticados, podem administrar integralmente recursos para materiais de consumo, materiais permanentes, serviços de terceiros e pessoal ${ }^{2}$.

O universo e o grau de autonomia financeira de estabelecimentos de saúde eram até o momento desconhecidos. O objetivo deste estudo foi descrever estabelecimentos de saúde brasileiros segundo esfera administrativa pública e privada, tipo de atendimento, região geográfica, natureza jurídica e grau de autonomia para administrar recursos financeiros. Esses compõem os primeiros resultados sobre administração financeira de estabelecimentos de saúde da Pesquisa de Assistência Médico-Sanitária do Instituto Brasileiro de Geografia e Estatística (AMS/ IBGE) de 2009.

\section{Método}

Foram utilizados microdados dos blocos 3 (caracterização do estabelecimento) e 25 (gestão de recursos financeiros) do censo nacional de estabelecimentos de saúde apresentado na AMS/ IBGE de 2009.

O grau de autonomia financeira (integral, parcial, nenhuma) foi descrito segundo: esfera administrativa (público federal, estadual, municipal e privado com e sem fins de lucro), tipo de atendimento (com ou sem internação e serviços de apoio diagnóstico e terapêutico) e localização geográfica (regiões Norte, Nordeste, Sudeste, Centro-oeste e Sul). Foi também analisado segundo as naturezas jurídicas, divididas em: administração pública (direta da saúde, educação e outros órgãos; autarquia; fundação pública); entidades sem fins de lucro (fundação privada, serviço social autônomo, organização social de interesse público, inclusive organização social de saúde, sindicato ou associação profissional); entidades empresariais (cooperativa; empresário individual; sociedades) e não classificados (outras associações e outros).

Autonomia financeira parcial foi definida como a autonomia para executar orçamento em pelo menos uma, mas não em todas as áreas administrativas (fundos para pequenas despesas emergenciais, materiais de consumo, materiais permanentes, serviços de terceiros, despesas com pessoal). Esfera administrativa define-se pela responsabilidade na gestão da operação do estabelecimento. Assim, organização social de interesse público e organização social de saúde, ainda que usem instalações da administração pública, são categorizadas como esfera administrativa privada sem finalidades de lucro.

Frequências absolutas e relativas das variáveis foram apresentadas na análise exploratória dos dados em IBM SPSS (IBM Corp., Armonk, Estados Unidos), com tabulações para grau de autonomia financeira de estabelecimentos de saúde segundo esfera administrativa, tipo de atendimento, grandes regiões (Tabela 1) e natureza jurídica (Tabela 2 ).

\section{Resultados}

Dos 97.449 estabelecimentos de saúde identificados na pesquisa, 52.055 se declararam da esfera administrativa pública e 45.394 da privada. Desses, apenas 5,2\% dos públicos e $8,7 \%$ dos privados não prestaram informações referentes à gestão de recursos financeiros (Tabela 1). Entre os estabelecimentos públicos, $772(1,5 \%)$ administravam seus próprios recursos financeiros 
Autonomia financeira de estabelecimentos de saúde, segundo esfera administrativa, tipo de atendimento e grandes regiões. Brasil, 2009.

\begin{tabular}{|c|c|c|c|c|c|c|c|c|c|}
\hline & \multicolumn{9}{|c|}{ Grau de autonomia financeira } \\
\hline & \multirow{2}{*}{$\begin{array}{c}\text { Total } \\
\mathbf{n}\end{array}$} & \multicolumn{2}{|c|}{ Integral } & \multicolumn{2}{|c|}{ Parcial } & \multicolumn{2}{|c|}{ Sem autonomia } & \multicolumn{2}{|c|}{ Sem resposta } \\
\hline & & $\mathbf{n}$ & $\%$ & $\mathbf{n}$ & $\%$ & $\mathbf{n}$ & $\%$ & $\mathbf{n}$ & $\%$ \\
\hline Públicos & 52.055 & 772 & 1,5 & 1.239 & 2,4 & 47.349 & 91,0 & 2.695 & 5,2 \\
\hline \multicolumn{10}{|l|}{ Esfera administrativa } \\
\hline Federal & 950 & 63 & 6,6 & 63 & 6,6 & 794 & 83,6 & 30 & 3,2 \\
\hline Estadual & 1.326 & 106 & 8,0 & 243 & 18,3 & 919 & 69,3 & 58 & 4,4 \\
\hline Municipal & 49.779 & 603 & 1,2 & 933 & 1,9 & 45.636 & 91,7 & 2.607 & 5,2 \\
\hline \multicolumn{10}{|l|}{ Tipo de atendimento } \\
\hline Com internação & 2.839 & 194 & 6,8 & 313 & 11,0 & 2.194 & 77,3 & 138 & 4,9 \\
\hline Sem internação & 47.425 & 494 & 1,0 & 876 & 1,8 & 43.615 & 92,0 & 2.440 & 5,1 \\
\hline SADT & 1.791 & 84 & 4,7 & 50 & 2,8 & 1.540 & 86,0 & 117 & 6,5 \\
\hline \multicolumn{10}{|l|}{ Grandes regiões } \\
\hline Norte & 4.577 & 27 & 0,6 & 84 & 1,8 & 4.367 & 95,4 & 99 & 2,2 \\
\hline Nordeste & 19.353 & 140 & 0,7 & 185 & 1,0 & 18.011 & 93,1 & 1.017 & 5,3 \\
\hline Centro-oeste & 3.909 & 32 & 0,8 & 26 & 0,7 & 3.721 & 95,2 & 130 & 3,3 \\
\hline Sudeste & 16.580 & 324 & 2,1 & 695 & 4,2 & 14.638 & 88,1 & 923 & 5,6 \\
\hline Sul & 7.636 & 249 & 3,3 & 249 & 3,3 & 6.612 & 86,6 & 526 & 6,8 \\
\hline Privados & 45.394 & 37.604 & 82,8 & 1.361 & 3,0 & 2.502 & 5,5 & 3.927 & 8,7 \\
\hline \multicolumn{10}{|l|}{ Esfera administrativa } \\
\hline Com fins de lucro & 41.375 & 34.702 & 83,9 & 1.075 & 2,6 & 1.925 & 4,7 & 3.673 & 8,9 \\
\hline Sem fins de lucro & 4.019 & 2.902 & 72,2 & 286 & 7,1 & 577 & 14,4 & 254 & 6,3 \\
\hline \multicolumn{10}{|l|}{ Tipo de atendimento } \\
\hline Com internação & 4.069 & 3.411 & 83,8 & 188 & 4,6 & 231 & 5,7 & 239 & 5,9 \\
\hline Sem internação & 20.874 & 17.445 & 83,6 & 610 & 2,9 & 954 & 4,6 & 1.865 & 8,9 \\
\hline SADT & 20.451 & 16.748 & 81,9 & 563 & 2,8 & 1.317 & 6,4 & 1.823 & 8,9 \\
\hline \multicolumn{10}{|l|}{ Grandes regiões } \\
\hline Norte & 1.829 & 1.686 & 92,2 & 40 & 2,2 & 74 & 4,0 & 29 & 1,6 \\
\hline Nordeste & 9.485 & 8.252 & 87,0 & 123 & 1,3 & 541 & 5,7 & 569 & 6,0 \\
\hline Centro-oeste & 4.710 & 4.146 & 88,0 & 156 & 3,3 & 184 & 3,9 & 224 & 4,8 \\
\hline Sudeste & 20.420 & 16.496 & 80,8 & 720 & 3,5 & 1.161 & 5,7 & 2.043 & 10,0 \\
\hline Sul & 8.950 & 7.024 & 78,5 & 322 & 3,6 & 542 & 6,1 & 1.062 & 11,9 \\
\hline
\end{tabular}

SADT: Serviços de Apoio Diagnóstico e Terapêutico.

Fonte: Pesquisa de Assistência Médico-Sanitária 2009 (AMS/IBGE).

integralmente, 1.239 (2,4\%) parcialmente e 91\% não administravam. Entre os estabelecimentos privados, $37.604(82,8 \%)$ administravam seus recursos integralmente, 1.361 (3\%) parcialmente e $5,5 \%$ não administravam (Tabela 1 ).

Na esfera administrativa pública, estabelecimentos estaduais apresentavam mais autonomia integral ou parcial $(26,3 \%)$, seguidos dos federais $(13,2 \%)$ e municipais $(3,1 \%)$. Considerando o tipo de atendimento, $17,8 \%$ dos estabelecimentos públicos com internação tinham algum grau de autonomia, sendo a autonomia parcial quase duas vezes mais frequente que a integral. Apenas em estabelecimentos públicos de apoio diagnós- tico e terapêutico (SADT) a autonomia integral $(4,7 \%)$ foi mais comum do que a parcial $(2,8 \%)$ (Tabela 1).

Quanto à região geográfica, estabelecimentos públicos do Centro-oeste $(1,5 \%)$ e Nordeste $(1,7 \%)$ tinham menos autonomia integral ou parcial do que os do Sul $(6,6 \%)$ e Sudeste $(6,3 \%)$. Nas regiões Norte, Nordeste e Sudeste predominou a autonomia parcial, e no Sul e Centro-oeste as autonomias integral e parcial se equivaleram (Tabela 1).

Na esfera administrativa privada, estabelecimentos privados com fins de lucro eram mais autônomos do que os sem fins de lucro $(83,9 \%$ 
Tabela 2

Estabelecimentos de saúde segundo grau de autonomia financeira e natureza jurídica. Brasil, 2009.

\begin{tabular}{|c|c|c|c|c|c|c|c|c|c|}
\hline \multirow[t]{3}{*}{ Natureza jurídica } & \multirow{3}{*}{$\begin{array}{c}\text { Estabelecimentos } \\
\text { n }\end{array}$} & \multicolumn{8}{|c|}{ Grau de autonomia financeira } \\
\hline & & \multicolumn{2}{|c|}{ Integral } & \multicolumn{2}{|c|}{ Parcial } & \multicolumn{2}{|c|}{ Sem autonomia } & \multicolumn{2}{|c|}{ Sem resposta } \\
\hline & & $\mathbf{n}$ & $\%$ & $\mathbf{n}$ & $\%$ & $\mathbf{n}$ & $\%$ & $\mathbf{n}$ & $\%$ \\
\hline Administração pública & 50.541 & 691 & 1,4 & 1.197 & 2,4 & 46.070 & 91,2 & 2.583 & 5,1 \\
\hline Administração direta em saúde (MS, SES, SMS) & 49.466 & 570 & 1,2 & 1.036 & 2,1 & 45.349 & 91,7 & 2.511 & 5,1 \\
\hline $\begin{array}{l}\text { Administração direta em educação (MEC, SEE, } \\
\text { SME) }\end{array}$ & 102 & 10 & 9,8 & 19 & 18,6 & 70 & 68,6 & 3 & 2,9 \\
\hline $\begin{array}{l}\text { Administração direta de outros órgãos } \\
\text { (Ministério da Defesa etc.) }\end{array}$ & 161 & 22 & 13,7 & 27 & 16,8 & 88 & 54,7 & 24 & 14,9 \\
\hline Autarquia & 308 & 43 & 14,0 & 48 & 15,6 & 188 & 61,0 & 29 & 9,4 \\
\hline Fundações públicas & 504 & 46 & 9,1 & 67 & 13,3 & 375 & 74,4 & 16 & 3,2 \\
\hline Entidades sem fins de lucro & 1.638 & 1.142 & 69,7 & 136 & 8,3 & 262 & 16,0 & 98 & 6,0 \\
\hline Fundações privadas & 492 & 340 & 69,1 & 36 & 7,3 & 103 & 20,9 & 13 & 2,6 \\
\hline $\begin{array}{l}\text { Organização social/Organização social de } \\
\text { interesse público }\end{array}$ & 525 & 356 & 67,8 & 45 & 8,6 & 84 & 16,0 & 40 & 7,6 \\
\hline Serviço social autônomo (SESI, SESC etc.) & 158 & 89 & 56,3 & 30 & 19,0 & 25 & 15,8 & 14 & 8,9 \\
\hline Sindicato ou associação profissional & 463 & 357 & 77,1 & 25 & 5,4 & 50 & 10,8 & 31 & 6,7 \\
\hline Entidades empresariais & 39.568 & 33.297 & 84,2 & 987 & 2,5 & 1.826 & 4,6 & 3.458 & 8,7 \\
\hline Cooperativa & 360 & 194 & 53,9 & 37 & 10,3 & 106 & 29,4 & 23 & 6,4 \\
\hline Empresário individual & 9.855 & 8.313 & 84,4 & 180 & 1,8 & 408 & 4,1 & 954 & 9,7 \\
\hline Sociedades & 29.353 & 24.790 & 84,5 & 770 & 2,6 & 1.312 & 4,5 & 2.481 & 8,5 \\
\hline Não classificados & 5.702 & 3.246 & 56,9 & 280 & 4,9 & 1.693 & 29,7 & 483 & 8,5 \\
\hline Outras associações & 1.568 & 1.187 & 75,7 & 90 & 5,7 & 167 & 10,7 & 124 & 7,9 \\
\hline Outros & 4.134 & 2.059 & 49,8 & 190 & 4,6 & 1.526 & 36,9 & 359 & 8,7 \\
\hline Total & 97.449 & 38.376 & 39,4 & 2.600 & 2,7 & 49.851 & 51,2 & 6.622 & 6,8 \\
\hline
\end{tabular}

MEC: Ministério da Educação e Cultura; MS: Ministério da Saúde; SEE: Secretarias estaduais de educação; SES: Secretarias estaduais de saúde; SESC: Serviço Social do Comércio; SESI: Serviço Social da Indústria; SME: Secretarias municipais de educação; SMS: Secretarias municipais de saúde.

Fonte: Pesquisa de Assistência Médico-Sanitária 2009 (AMS/IBGE).

vs. 72,2\%). Quase não houve diferenças segundo tipo de atendimento. Para a região geográfica, Centro-oeste, Nordeste e Norte apresentaram maior frequência de autonomia do que Sul e Sudeste, invertendo-se o padrão observado na esfera administrativa pública.

Quanto à natureza jurídica (Tabela 2), 3,8\% dos estabelecimentos da administração pública e $78 \%$ das entidades sem fins de lucro referiram algum grau de autonomia. Empresas e sociedades privadas tiveram mais autonomia do que todas as demais naturezas jurídicas $(86,7 \%)$, predominando a autonomia integral.

Na administração pública direta da saúde (Tabela 2), 570 estabelecimentos declararam autonomia financeira integral e 1.036 referiram autonomia parcial, totalizando $3,3 \%$ destes estabelecimentos. Autonomia parcial foi mais frequente $(2,1 \%)$ que a integral $(1,2 \%)$. Em outras naturezas jurídicas da administração pública, a frequência de qualquer grau de autonomia foi bem maior - variou de 22,4\% (fundações públicas) a 30,5\% (administração direta de órgãos da defesa e outros).

Apesar da baixa frequência relativa de autonomia financeira entre estabelecimentos públicos da administração direta da saúde, o número absoluto daqueles com autonomia (1.606) superou o de outras naturezas jurídicas de administração pública e entidades sem fins de lucro com autonomia (1.560).

Recusas e outras não respostas foram baixas (3\% a 15\%) e mais frequentes entre órgãos militares da administração direta (14,9\%).

\section{Discussão}

Este é o primeiro trabalho nacional a dimensionar a autonomia financeira de estabelecimentos de saúde segundo natureza jurídica e região geográfica. Um estudo anterior 2 analisou a au- 
tonomia para gestão orçamentária em hospitais públicos das regiões metropolitanas do Rio de Janeiro e São Paulo, detalhando aspectos qualitativos.

Uma das forças deste trabalho é o baixo índice de não respostas. O único estudo brasileiro que buscou informação financeira em ampla escala em estabelecimentos de saúde tinha um universo de 2.258 hospitais. Para informações financeiras detalhadas, com 102 entidades pesquisadas, as perdas foram de $24,3 \% 5$.

Os resultados confirmam um espectro crescente de autonomia vinculado à natureza jurídica. No extremo com menor autonomia estão os estabelecimentos da administração direta da saúde e, no mais autônomo, entidades empresariais. Entre os dois, sucedem-se estabelecimentos da administração direta não saúde, com frequência de autonomia semelhante a autarquias e fundações públicas, e entidades sem fins de lucro.

Estudos internacionais vêm discutindo os impactos da autonomia em estabelecimentos públicos, principalmente no âmbito hospitalar 6,7, em que também no Brasil identifica-se a maior prevalência de autonomia pública. Para Paim ${ }^{8}$, baixa autonomia financeira na administração direta da saúde é uma questão central no SUS, e ainda hoje pode atingir inclusive órgãos gestores, como secretarias de saúde. Os resultados deste estudo confirmam que ela é especialmente crítica em estabelecimentos municipais, onde reside hoje a responsabilidade pela maior parte da prestação de serviços no país.

O expressivo crescimento de estabelecimentos simplificados, pela expansão da Estratégia Saúde da Família (ESF), pode ser invocado como motivo da menor autonomia financeira municipal frente a outros níveis de gestão, mas não parece ser a única explicação. Apesar de as coberturas da ESF nas regiões Centro-oeste, Norte e Sul à época da AMS/IBGE serem semelhantes (Departamento de Atenção Básica, Ministério da Saúde. Histórico de cobertura da saúde da família. http://dab.saude.gov.br/portaldab/historico_ cobertura_sf.php, acessados em 08/Jul/2013), a autonomia de estabelecimentos públicos era de $1,5 \%$ e $2,4 \%$ nas duas primeiras, mas de $6,6 \%$ no Sul. Isso sugere uma base de decisão política local favorável à maior autonomia financeira, que não necessariamente se restringe a mudanças de natureza jurídica dos estabelecimentos de saúde, mas que pode incluí-las. A incorporação dessas novas naturezas jurídicas, especialmente as organizações sociais de saúde, é mais intensa na Região Sudeste, que já mostrava um percentual elevado de estabelecimentos públicos com autonomia financeira neste estudo.

A principal limitação deste trabalho prendese ao caráter autoclassificatório das respostas. Principalmente para a natureza jurídica, isso pode ter permitido alguns erros de classificação. Entretanto, a magnitude de perdas de informação não compromete a generalização dos resultados.

Conclui-se que um número não desprezível de estabelecimentos de saúde da administração direta tem autonomia financeira, ainda que, em estabelecimentos de outras naturezas jurídicas ela seja mais comum. A AMS/IBGE 2009 pode viabilizar novas análises sobre a associação entre autonomia financeira e aspectos estruturais dos serviços de saúde, que merecem ser exploradas em estudos futuros.

\section{Resumen}

La autonomía financiera es un diferencial positivo en la administración pública, que se menciona en la Encuesta Nacional de Medicina y Salud (2009) (AMS/ IBGE) de Brasil en un 3,9\% de los 52.055 establecimientos públicos de salud. Fue más frecuente en hospitales públicos (17,8\%), gobiernos provinciales (26,3\%), el Sur $(6,6 \%)$ y, en general, fue parcial (sólo en algunas áreas, como gastos de emergencia, suministros, bienes de capital, servicios subcontratados y personal). La naturaleza jurídica de un 74,3\% de las 2264 instalaciones públicas que reportaron alguno grado de autonomía fue la administración directa. La concesión de autonomía financiera en la salud pública parece estar ligada a po- líticas locales y no se refiere necesariamente a cambios en la naturaleza jurídica. Sin embargo, se confirmó un espectro creciente de autonomía financiera, según la naturaleza jurídica, siendo los establecimientos de la administración directa de la salud menos autónomos frecuentemente y las sociedades empresariales más autónomas; el 85,8\% de los 45.394 establecimientos privados tenían autonomía financiera.

Administración de los Servicios de Salud; Administración Financiera; Investigación sobre Servicios de Salud 


\section{Colaboradores}

M. A. B. Santos participou da concepção do trabalho, análise dos dados, busca bibliográfica, elaboração do texto, organização, revisão crítica e aprovação final do artigo. F. C. Madeira colaborou na elaboração do texto, organização, análise dos dados e aprovação final do artigo. S. R. L. Passos e M. A. R. Andreazzi contribuíram na concepção do trabalho, análise dos dados, revisão crítica e aprovação final do artigo. F. Bakr e K. B. Oliveira participaram na análise dos dados e revisão crítica do artigo.

\section{Nota}

As opiniões, informações, dados e conceitos contidos neste trabalho são de exclusiva responsabilidade dos autores, não cabendo ao Instituto Brasileiro de Geografia e Estatística nenhuma responsabilidade por eles.

\section{Agradecimentos}

Este trabalho contou com o auxílio do Programa de Apoio à Pesquisa, Desenvolvimento e Inovação em Saúde Pública da Escola Nacional de Saúde Pública Sergio Arouca, Fundação Oswaldo Cruz (Inova-ENSP), projeto ENSP-013-LIV-10-2-10, Avanços para a Conta-Satélite de Saúde. S.R.L.P. é bolsista JCNE da FAPERJ.

\section{Referências}

1. Mazzilli HN. Manual do promotor público. São Paulo: Saraiva; 1991.

2. Ribeiro JM, Costa NR, Silva PLB. Inovações na gestão descentralizada de redes e organizações hospitalares: os casos das Regiões Metropolitanas do Rio de Janeiro e São Paulo In: Negri B, di Giovanni G, organizadores. Brasil: radiografia da saúde. Brasília: Ministério da Saúde; 2001. p. 554-78.

3. Vilasbôas ALQ, Paim JS. Práticas de planejamento e implementação de políticas no âmbito municipal. Cad Saúde Pública 2008; 24:1239-50.

4. Bresser-Pereira LC. Um novo Estado para a América Latina. Novos Estudos CEBRAP 1998; (50):91-8.

5. Portela MC, Barbosa PR, Ugá MAD, Lima SML, Gerschman SV. Hospitais filantrópicos no Brasil. Rio de Janeiro: Banco Nacional do Desenvolvimento; 2002. (BNDES Social, 5).
6. Allen P, Keen J, Wright J, Dempster P, Townsend J, Hutchings A, et al. Investigating the governance of autonomous public hospitals in England: multisite case study of NHS foundation trusts. J Health Serv Res Policy 2012; 17:94-100.

7. Casasnovas GL, McDaid D, Costa-Font J. Decentralization and management autonomy? Evidence from the Catalonian hospital sector in a decentralized Spain. International Public Management Review 2009; 10:103-19.

8. Paim JS. Aspectos críticos da institucionalização da planificação em organizações públicas de saúde. In: Paim JS, organizador. Saúde: política e reforma sanitária. Salvador: Instituto de Saúde Coletiva, Universidade Federal da Bahia; 2002. p. 407-43.

Recebido em 06/Mar/2013

Versão final reapresentada em 26/Jul/2013

Aprovado em 27/Set/2013 